\title{
MedienPädagogik
}

Zeitschrift für Theorie und Praxis der Medienbildung

Jahrbuch Medienpädagogik 4.

Zweitveröffentlichung aus: Jahrbuch Medienpädagogik 4. (2005) Wiesbaden: VS Verlag für Sozialwissenschaften. Hrsg. v. Ben Bachmair, Peter Diepold und Claudia de Witt.

\section{Die Bedeutung der (Allgemeinen) Didaktik für das Lehren und Lernen in virtuellen Räumen}

\author{
Bardo Herzig
}

Im vorliegenden Beitrag ${ }^{1}$ nehme ich die Frage auf, welcher Stellenwert der Allgemeinen Didaktik im Kontext von Lehren und Lernen in virtuellen Räumen zukommt. Dazu gehe ich zunächst von Präsenzsituationen aus und skizziere eine Vorstellung von entsprechenden Lehr- und Lernprozessen. Ein besonderes Augenmerk wird dabei auf die handlungsund entwicklungsorientierte Gestaltung dieser Prozesse gerichtet. Ausgehend von der skizzierten Modellvorstellung, wird im nächsten Schritt diskutiert, welche Veränderungen und didaktischen Konsequenzen sich ergeben, wenn Lernprozesse nicht mehr in realen Räumen mit sozialer Präsenz stattfinden, sondern in der Auseinandersetzung mit virtuellen oder teilvirtuellen Angeboten. Abschließend werden die Überlegungen auf das Beispiel des Fernstudiengangs Medien (FESTUM) bezogen und Problemlagen und Perspektiven aus didaktischer Sicht diskutiert.

\section{Lehr- und Lernprozesse als soziale Interaktion}

\section{Allgemeine Strukturmomente}

Lehr- und Lernprozesse fasse ich - im Sinne einer Modellvorstellung für Unterricht - zunächst als Interaktion von Lernenden und Lehrperson auf (vgl. Tulodziecki 1996, S. 132 ff.): Die Lernenden kommen mit bestimmten Lernvoraussetzungen in den LehrLernprozess - d.h. den Unterricht - und führen bestimmte Lernaktivitäten aus, die gewisse Lerneffekte haben. Die Lehrperson hat bestimmte Zielvorstellungen, realisiert entsprechende Lehrhandlungen und bildet auf Grund der Lernaktivitäten der Lernenden gewisse Annahmen zum Lernerfolg, die zu einer Modifizierung der Lehrhandlungen führen können. Lernaktivitäten sind dabei stets an bestimmte Inhalte, Erfahrungsformen bzw. Medien und Sozialformen gebunden. Als weitere Rahmenbedingungen von Lehr- und Lernprozessen sind - soweit es sich nicht

1 Für kritische Anmerkungen und konstruktive Anregungen zum Beitrag danke ich Prof. Werner Sesink. 
um Einzellernen handelt - die Lerngruppe, der institutionelle Kontext - z.B. Schule, Weiterbildungseinrichtung, Universität o.ä. - und die gesellschaftlichen Rahmenbedingungen - z.B. verfassungsbezogene Grundlagen oder bildungspolitische Beschlüsse - zu sehen (vgl. Abb. 1).

\section{Medien als Strukturmoment}

Der Ausweis von Medien als ein Strukturmoment von Unterricht in dieser Modellvorstellung ist in der Allgemeinen Didaktik nicht seit jeher selbstverständlich. Lange Zeit wurden sie in der Didaktik nur als (bloße) Hilfsmittel im Kontext methodischen Vorgehens gesehen. Spätestens aber mit der Entwicklung des Fernsehens und seit den didaktischen Überlegungen von Heimann (1962) werden sie als eigenständiges Strukturelement von Lehren und Lernen betrachtet. Dies bedeutet insbesondere, dass Medien in ihrer Wechselwirkung zu anderen Strukturelementen von Lehren und Lernen gesehen werden müssen. Die Stellung der Medien zwischen Lehrhandlungen und Lernaktivitäten macht deutlich, dass es sich bei den Medien um eine besondere Form der Erfahrung in der Auseinandersetzung mit einem Lerngegenstand handelt. Solche Erfahrungsformen - als Art und Weise, wie ein Lernender mit einem Lerngegenstand in Beziehung tritt - können real, modellhaft, abbildhaft oder symbolisch sein (vgl. Tulodziecki 1997, S. 34). Die unterrichtliche Auseinandersetzung mit z.B. dem Wattenmeer könnte demnach in unmittelbarer Begegnung, über ein Modell, mit Hilfe von Fotos, Diagrammen oder Schaubildern, Filmen und Animationen oder über Texte und Vorträge erfolgen.

Im Kontext der nachfolgenden Überlegungen ist es zweckmäßig, den Medienbegriff auf abbildhafte und symbolische Erfahrungsformen einzugrenzen und nur solche Artefakte als Medien zu bezeichnen, bei denen die Präsentation, Speicherung, Übermittlung, Verarbeitung oder Verknüpfung von Zeichenaspekten mit technischer Unterstützung geschieht (vgl. Herzig 2002, S. 287 ff.). 


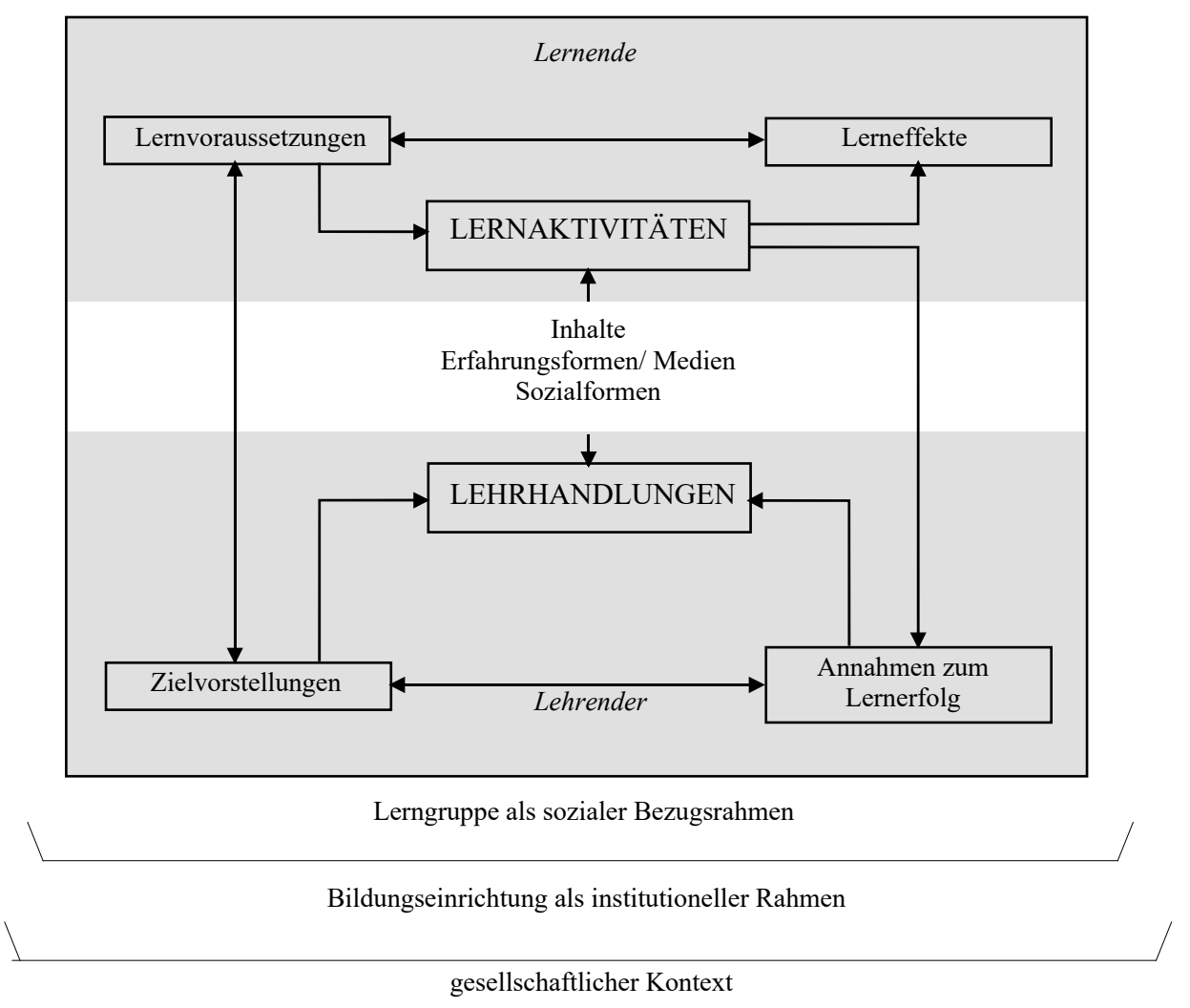

Abb. 1: Modellvorstellung für Lehren und Lernen (vgl. Tulodziecki 1996, S. 135).

\section{Allgemeine Zielvorstellungen}

Die allgemein didaktische Diskussion hat auch auf die Bestimmung von Zielvorstellungen von Unterricht Einfluss genommen. So finden sich z.B. bei Klafki Forderungen nach Selbstbestimmungs-, Mitbestimmungs- und Solidaritätsfähigkeit (vgl. 1985), bei Schulz (1981) Forderungen nach Kompetenz, Autonomie und Solidarität oder bei Winkel (1983) nach Demokratisierung und Humanisierung. Darüber hinaus wird in aktuellen didaktischen Konzepten Handlungsorientierung als Prinzip hervorgehoben (vgl. z.B. Jank/ Meyer 1991; Gudjons 1992; Tulodziecki 1996). Mit solchen Überlegungen ist u.a. auch die Tendenz verbunden, allgemeine Ziele nicht so sehr inhaltlich festzulegen, sondern eher als Schlüsselqualifikationen oder als allgemeine Kompetenzen zu formulieren (vgl. Bunk/ Kaiser/ Zedler 1991). Ein solches Verständnis von allgemeinen Zielen im Kontext von Lehren und Lernen liegt auch diesem Beitrag zugrunde. Zusammenfassend kann von der allgemeinen Zielvorstellung eines sachgerechten, selbstbestimmten, kreativen Handelns in sozialer Verantwortung gesprochen werden (vgl. auch Tulodziecki 1993, 
BLK 1995)². Damit sind zum einen fachspezifische Zielvorstellungen eingeschlossen, zum anderen ist deutlich gemacht, dass Lehr- und Lernprozesse in institutionalisierten Zusammenhängen für gegenwärtiges und zukünftiges Handeln bedeutsam sein sollen.

Ausgehend von einer solchen Auffassung von Lehr- und Lernprozessen, sind im Hinblick auf den Stellenwert einer Allgemeinen Didaktik in virtuellen Räumen zunächst folgende weiteren Fragen zu klären:

- Wie können Lernprozesse angemessen angeregt und unterstützt werden?

- Welche Besonderheiten ergeben sich, wenn Lehr- und Lernprozesse nicht in Präsenzszenarien stattfinden?

\section{Lernen als Handlungsprozess}

Lern- und Handlungsmodell

Im Anschluss an die Beschreibung von Lehr- und Lernprozessen auf der Ebene sozialer Interaktionen kann Lernen - aus der Sicht des Individuums - auch als ein Handlungsprozess verstanden werden, der auf die Erweiterung des (themenspezifischen) Wissens, des Erfahrungsstandes sowie die (Weiter-)Entwicklung der intellektuellen Fähigkeiten und der Werthaltungen ausgerichtet ist (vgl. Abb. 2). Eine solche Vorstellung steht im Einklang mit der o.g. Zielvorstellung eines sachgerechten, selbstbestimmten, kreativen und sozial verantwortlichen Handelns.

Zur Erläuterung des Modells sei angenommen, dass eine Studentin ein Seminar zu Lese-Rechtschreib-Schwierigkeiten besucht, dessen Zielsetzung darin besteht, Diagnosefähigkeiten zu entwickeln und konkrete Fördermöglichkeiten für betroffene Kinder in der Grundschule zu erarbeiten. Diese Anforderung aktiviert bei der Studentin das Bedürfnis, ihre bestehende Unsicherheit in diesem Bereich abzubauen sowie sich selbst als kompetent zu erleben und entsprechende Anforderungen in der Zukunft bewältigen zu können. Mit anderen Worten: Die Problemstellung des Seminars wird als bedeutsam im Hinblick auf die zukünftige Lebenssituation empfunden. Diese Wechselbeziehung zwischen situativer Anforderung und aktivierten Bedürfnissen lässt sich psychologisch als Motivation deuten, die dazu führt, dass die Studentin im Laufe des Seminars in der Auseinandersetzung mit einzelnen Fragestellungen entsprechende Lernaktivitäten ausführt. Auf die Lernaktivitäten selbst nehmen zum einen das bisherige Wissen und

2 Eine solche Formulierung bedarf im Grunde einer ausgiebigen Reflexion, z.B. im Hinblick auf das zugrunde liegende Bildungsverständnis. Die hier vorgestellte - normative - Bestimmung einer allgemeinen Zielvorstellung von Lehr- und Lernprozessen soll nicht darüber hinwegtäuschen, dass Allgemeine Didaktik - wenngleich sie heute vielfach im Mainstream auf eher lerntheoretische Fragen zugespitzt wird - nicht auf methodische Fragen verkürzt werden darf, sondern auch weiterhin - allerdings unter veränderten Rahmenbedingungen nach dem Allgemeinen in Bezug auf das Kulturelle, das Soziale, das Anthropologische usw. fragen muss. In bezug auf die Medien bedeutet dies nicht zuletzt auch die Reflexion ihres allgemeinbildenden Wertes neben ihren vermittlungsrelevanten didaktischen Eigenschaften (vgl. Herzig 2002, S. 296 ff.). 
die vorhandenen Erfahrungen Einfluss, zum anderen die intellektuellen Fähigkeiten und die Werthaltungen. Das Wissen lässt sich dabei noch einmal in verschiedene Kategorien unterscheiden, z.B. Alltagswissen oder Expertisewissen, bereichsspezifisches oder allgemeines Wissen, Strategiewissen oder Kontrollwissen usw.. Die intellektuellen Fähigkeiten können als Ausdruck der individuellen kognitiven Komplexität verstanden werden, d.h. als Grad der Fähigkeit, unterschiedliche Perspektiven in den Blick zu nehmen, kriterienbezogen auf unterschiedlichen Abstraktionsniveaus zu denken und zu argumentieren sowie verschiedene Aspekte in komplexen Gefügen zu verknüpfen (vgl. Schroder/ Driver/ Streufert 1975; Streufert/ Streufert 1978, S. 17 ff.). Diese intellektuellen Fähigkeiten sind bereichsunspezifisch. Die Wertvorstellungen lassen sich vor dem Hintergrund des Ansatzes von Kohlberg (vgl. 1974; 1977) als Stufen von Gerechtigkeits- und Fairnessvorstellungen oder - mit Bezug auf den Ansatz von Gilligan (1991) als verantwortungs- und empathiebezogene Vorstellungen interpretieren. Die Durchführung der Lernaktivitäten wird sich in einem Lernergebnis niederschlagen, das seinerseits bestimmte Rückwirkungen auf die Bedürfnislage, den Wissensstand sowie die intellektuellen Fähigkeiten und die Werthaltungen hat. Stellt die Studentin beispielsweise fest, dass sie im Verlauf des Seminars Wissen erworben hat und Erfahrungen sammeln konnte, die ihr im Umgang mit lese-rechtschreibschwachen Kindern helfen, kann dies zur Befriedigung der ursprünglich angeregten Bedürfnisse führen. Darüber hinaus können die Lernaktivitäten zu einer Veränderung z.B. der Werthaltungen führen, indem die Studentin sich mit einem defizitorientierten Ansatz zu Legasthenie beschäftigt hat und diesen als eine nicht akzeptable und nicht der Person des Kindes gerecht werdende Beschreibung von Lese-Rechtschreib-Schwierigkeiten kennen gelernt hat.

An dieser Stelle wird deutlich, dass die Auseinandersetzung mit bedeutsamen Aufgabenstellungen in einem bestimmten Kontext nicht dazu führen darf, den Kontext selbst einer kritischen Reflexion zu entziehen. Mit anderen Worten, die Lösung von Problemen, das Treffen von Entscheidungen oder die Beurteilung von Fällen im Rahmen von handlungsorientierten Lehr- und Lernprozessen muss selbst immer wieder der Frage unterworfen werden, ob dieser Kontext didaktisch angemessen und legitimiert ist. Eine fraglose Anerkennung eines bestimmten Kontextes - etwa in Aufgaben als bedeutsam herausgestellter schulischer Situationen - oder eines didaktischen Modells widerspräche letztlich auch dem Modell zugrunde liegenden Prozess des Erwägens und dem Ziel eines selbstbestimmt Lernenden (vgl. auch Abschn. 5). 


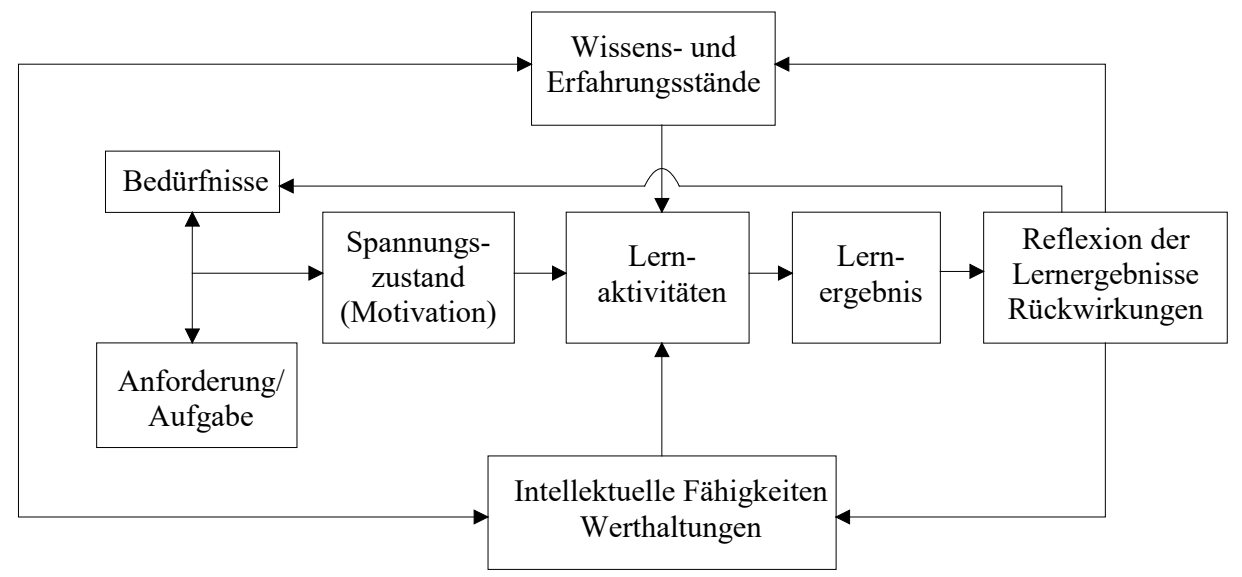

Abb. 2: Lern- und Handlungsmodell.

Die hier skizzierte Modellvorstellung vom Lernen stellt gleichzeitig eine Beschreibung allgemeiner (menschlicher) Handlungsprozesse dar. In der allgemeineren Form würde es im Kern nicht um Lernaktivitäten, sondern um die Auswahl und Bewertung von Handlungsmöglichkeiten ${ }^{3}$ gehen, die Lernergebnisse wären entsprechend Handlungsfolgen, die reflektiert werden und zu entsprechenden Rückwirkungen auf die Bedingungsfaktoren (Bedürfnisse Wissen, Erfahrung, intellektuelles Niveau, Wertvorstellungen) führen.

\section{Lerntheoretische Aspekte}

Die zuvor entwickelte Vorstellung vom Lernen enthält implizite Annahmen über Lernprozesse, wie sie auch in verschiedenen lerntheoretischen Ansätzen zu finden sind. Die Grundannahme besteht in diesem Fall darin, dass Lernen ein aktiver Prozess des Individuums in der Auseinandersetzung mit der Umwelt ist, der dem Ziel einer Ausdifferenzierung, Erweiterung oder Veränderung von Entwicklungsständen und kognitiven Strukturen dient (s.o.). Dieser Prozess findet auf der Basis des Vorwissens - oder allgemeiner: des jeweiligen Erfahrungs- und Kenntnistandes sowie des Standes der sozial-kognitiven Entwicklung - statt. Damit sind sowohl kognitionstheoretische als auch konstruktivistische Aspekte angesprochen. Die konstruktivistischen Ansätze stellen heute den theoretisch bedeutsamsten Rahmen zur Beschreibung von Lernprozessen dar. Allerdings sind die Spielarten und Varianten so zahlreich, dass von einer einheitlichen Theorie nicht gesprochen werden kann. Wertet man die verschiedenen Lesarten aus, so lassen sich erkenntnistheoretische, kognitive und soziale Akzentuierungen unterscheiden (vgl. z.B. Schnotz 2003). Auf die didaktische Diskussion haben insbesondere Ansätze des situ-

3 Vom Handlungsbegriff ausgeschlossen sind hier Routinen, die in einer spezifischen Situation keiner Auswahl von Alternativen mehr gegenübergestellt werden, und angemessener durch den Verhaltensbegriff charakterisiert sind. 
ierten Lernens Einfluss gewonnen, die zu den kognitivistisch und sozial ausgerichteten Varianten des Konstruktivismus zählen. Sie gehen von folgenden Annahmen aus (vgl. Mandl/ Gruber / Renkl 2002, S. 142):

- Wissen wird durch das wahrnehmende Subjekt konstruiert und ist situiert, d.h. es ist gebunden an die Situation, in der es erworben wurde,

- Lernen ist ein sozialer Prozess, in dem insbesondere gesellschaftlich geteiltes Wissen erworben wird,

- Wissen wird unter dem Aspekt seiner Anwendungsfähigkeit, d.h. seiner Authentizität analysiert: Lernprozesse sollen dem Erwerb flexiblen Wissens dienen und kein träges Wissen hervorbringen.

Didaktisch sind diese Annahmen insofern mit weitreichenden Konsequenzen verbunden, als die Annahme einer „Übertragung“ von Wissen - im Sinne objektiv vorhandener Wissensbestände - damit nicht mehr haltbar ist. Darüber hinaus gilt es, Wissen in solche Kontexte einzubetten, in denen es zur Anwendung kommen kann. Da der Erwerb des Wissens zunächst an die jeweilige Erwerbssituation gebunden ist, müssen Möglichkeiten des Transfers didaktisch gefördert und gefordert werden.

\section{Konsequenzen}

Auf der Basis der bisherigen Überlegungen lässt sich aus didaktischer Sicht zunächst Folgendes festhalten:

- Leitideen für Lehren und Lernen sollten ein sachgerechtes, selbstbestimmtes, kreatives und sozialverantwortliches Handeln sein.

- Ein solches Handeln setzt bei Lehr- und Lernprozessen die Beachtung von Lebenssituation und Bedürfnislage der Lernenden voraus und macht es erforderlich, beim Erwerb neuen Wissens und neuer Erfahrungen zugleich die intellektuelle und wertbezogene Entwicklung zu fördern.

Darüber hinaus sollte für den Lernprozess selbst Folgendes gelten:

- Lehren und Lernen sollen jeweils von einer - für die Lernenden bedeutsamen - Aufgabe ausgehen. Solche Aufgaben können z.B. Problemstellungen, Entscheidungsfälle, Gestaltungs- und Beurteilungsaufgaben sein.

- Lehren und Lernen sollen darauf gerichtet sein, vorhandenes Wissen oder bestehende Fertigkeiten zu einem Themengebiet zu aktivieren und - von dort ausgehend - eine Korrektur, Erweiterung, Ausdifferenzierung oder Integration von Wissen und Vorstellungen zu erreichen.

- Lehren soll eine aktive und kooperative Auseinandersetzung der Lernenden mit einer Aufgabe ermöglichen, indem - auf der Basis geeigneter Informationen - selbstständig Lösungswege entwickelt und erprobt werden.

- Lehren soll den Vergleich unterschiedlicher Lösungen ermöglichen sowie eine Systematisierung und Anwendung angemessener Kenntnisse und Vorgehensweisen sowie deren Weiterführung und Reflexion. 


\section{Zur Anregung und Unterstützung von Lernprozessen}

\section{Initiierung von Lernprozessen}

Aus der Modellvorstellung zum Handeln bzw. zum Lernen ergibt sich die Forderung, Lernprozesse durch geeignete Aufgabenstellungen anzuregen. Solche Aufgaben sind für Lernende insbesondere dann lern- und entwicklungsfördernd, wenn sie erstens auf ein Bedürfnis und Interesse bezogen werden können und damit Bedeutsamkeit für die Lernenden erlangen und zweitens einen Neuigkeitswert für die Lernenden besitzen - also nicht mit vorhandenen Kenntnissen gelöst werden können -, zugleich aber die Chance auf ihre Bewältigung bieten, d.h. einen angemessenen Schwierigkeitsgrad aufweisen. Aus didaktischer Sicht ist es darüber hinaus wünschenswert, dass die Aufgaben es ermöglichen, einen Lerninhalt exemplarisch zu erschließen und in orientierendes Lernen einzumünden, sodass einerseits - angesichts der Vielfältigkeit von Informationen - eine angemessene Tiefe der Auseinandersetzung erreicht wird und andererseits eine ordnende Übersicht entstehen kann.

Die lerntheoretischen Überlegungen lassen es zudem geboten erscheinen, komplexe Ausgangsprobleme zu wählen, einen Rahmen und Anwendungskontext für Wissen bereitzustellen, multiple Perspektiven auf Problemstellungen oder Aufgaben anzubieten, Lernprozesse zu artikulieren und zu reflektieren sowie kooperatives Arbeiten in Gruppen oder mit Experten zu ermöglichen (vgl. Mandl/ Gruber/ Renkl 2002, S. 143 f.). Das komplexe Ausgangsproblem soll ein „interessantes und intrinsisch motivierendes Problem“ darstellen, das möglichst realistisch ist, d.h. möglichst all die strukturellen und inhaltlichen Merkmale aufweist, wie sie typisch für die reale Situation sind, in der das Problem (oder die Gestaltungs- und Beurteilungsaufgabe bzw. der Entscheidungsfall) angesiedelt ist. Mit Bezug auf die einführenden Überlegungen bedeutet dies, dass durch die Aufgabenstellung relevante Bedürfnisse angesprochen werden und die Aufgabe für die gegenwärtige oder zukünftige Lebenssituation des Lernenden Bedeutsamkeit aufweist. Die Einnahme multipler Perspektiven soll die Verankerung des Wissens in einem isolierten Kontext lösen und flexible Anwendung fördern. Diesem Ziel dienen auch die Artikulation und Reflexion von Lernwegen und Lösungsprozessen.

\section{Weiterführender Ablauf von Lernprozessen}

Auf der Basis der obigen generellen Forderungen an Lehr-Lernprozesse lassen sich für ein handlungs- und entwicklungsorientiertes Vorgehen - unter Berücksichtigung kognitionsorientierter und konstruktivistischer Annahmen - insgesamt folgende Phasen als idealtypische Strukturierung von Lehr-Lernprozessen vorschlagen (vgl. Tulodziecki 1996, S. 128 ff.): 
- Aufgabenstellung, Sammeln und Problematisieren spontaner Lösungsvermutungen,

- Zielfestlegung und Besprechen der Bedeutsamkeit,

- Verständigung über das Vorgehen,

- Erarbeitung von Grundlagen für die Aufgabenlösung,

- Durchführung der Aufgabenlösung,

- Vergleich von Lösungen und Zusammenfassung des Gelernten,

- Einführen von Anwendungsaufgaben und deren Bearbeitung,

- Weiterführung und Reflexion des Gelernten und der Lernwege.

Solche Phasen lassen sich zugleich als Weiterführung der didaktischen Diskussion um eine angemessene Strukturierung von Unterricht oder Projektarbeit - von Herbart über Kerschensteiner und Roth bis Gudjons - deuten. Die Phasen sind dabei nicht als starre Formalstufen, sondern als flexibles Grundmuster für Lehr- und Lernprozesse zu verstehen.

Mit der Forderung nach einem problem-, entscheidungs-, gestaltungs- und beurteilungsorientierten Vorgehen ist zugleich die Annahme verbunden, dass so - unter Beachtung von Bedürfnissen und Lebenssituationen - ein angemessener Wissens- und Erfahrungsstand bei gleichzeitiger Förderung des intellektuellen und sozial-moralischen Entwicklungsniveaus erreicht werden kann. Dabei sind auch Fähigkeitsbereiche angesprochen, die für die Bildung in einer durch Medien und Informationsfülle gekennzeichneten Zeit besonders bedeutsam sind, und zwar Problemlösefähigkeit, Entscheidungsfähigkeit, Gestaltungsfähigkeit und Urteilsfähigkeit.

Nach dieser Auseinandersetzung mit Lehr- und Lernprozessen und den daraus formulierten allgemein didaktischen Anforderungen soll im Folgenden diskutiert werden, welche Veränderungen sich ergeben, wenn das traditionelle Setting eines Lehr-Lernprozesses verlassen wird und zunehmend Medien Funktionen im Lehr- und Lernprozess übernehmen.

\section{Zur Rolle der Medien in Lehr- und Lernprozessen}

\subsection{Von realen zu virtuellen Räumen}

Ich habe in der allgemeinen Modellvorstellung von Lehr- und Lernprozessen (vgl. Abschn. 1) Medien als einen konstitutiven Bestandteil von Lehr- und Lernprozessen ausgewiesen und betont, dass Medien in Wechselbeziehung zu anderen Strukturmomenten zu sehen sind. Diese Vorstellung geht zunächst einmal davon aus, dass Lehrende und Lernende in interfazialen Settings miteinander lernen, d.h. sich in realen Räumen befinden.

Prinzipiell kann an die Stelle einer Lehrperson aber auch ein Lehrsystem treten, z.B. ein Online-Angebot, das die Funktion der Lernanregung und Lernunterstützung im Sinne von Lehrhandlungen übernimmt. Dabei variiert der Grad der Übernahme von Lehraktivitäten. Die Pole bilden „didaktisch ge- 
schlossene“ Angebote - z.B. im Sinne von CBTs zu einem bestimmten Themenbereich - bzw. „didaktisch offene“ Angebote - z.B. Arbeits- und Kooperationsplattformen mit bestimmten Groupware-Funktionalitäten oder Softwarewerkzeugen, wie z.B. ein Grafik- oder Textverarbeitungsprogramm. Das Extrem der geschlossenen Angebote ist inhaltsgebunden und enthält eine „implementierte Didaktik“, d.h. möglichst viele Lehrfunktionen sind in das Angebot selbst verlagert, eine Betreuung oder eine Einbettung in soziale Kontexte ist nicht vorgesehen. Das andere Extrem stellen inhaltsneutrale Angebote dar, die über medienspezifische Funktionalitäten - z.B. Kommunikationstools, Visualisierungswerkzeuge, Dokumentenverwaltungen usw. - verfügen, d.h. die didaktische Gestaltung des Lernprozesses ist nicht im Vorhinein festgelegt, sondern wird durch die Art der Nutzung des Mediums bestimmt. ${ }^{4}$

Nicht jedes computerbasierte Medienangebot, in dem bestimmte Lehrfunktionen realisiert sind, wird - insbesondere wenn es sich um Offline-Angebote handelt - im allgemeinen Sprachgebrauch als virtuelles Angebot bzw. die Auseinandersetzung damit als Lernen in virtuellen Räumen bezeichnet. Insofern scheint es geboten, zunächst eine begriffliche Klärung zu versuchen.

Orientiert man sich an der traditionellen Raumauffassung im Kontext von Lehren und Lernen, so ist zunächst ein Klassenraum, ein Vorlesungssaal oder ein Seminarraum angesprochen, der einen Ort der Versammlung darstellt, an dem dann unter Anwesenheit der Versammelten Lehr- und Lernaktivitäten durchgeführt werden können. Solche Räume sind in der Regel durch bestimmte Ausstattungsmerkmale als Lernorte erkennbar, z.B. durch die Ausstattung mit Schreibtischen und Stühlen, ggf. einzelnen Medien, wie z.B. Tafel, Overheadprojektor oder Flipchart. Mitunter lässt auch die architektonische Gestaltung schon Rückschlüsse auf bestimmte Lernaktivitäten oder Sozialformen zu, z.B. die Anordnung der Stühle und Tische, etwa in U-Form, uniform ausgerichteten Reihen oder in Form von kleinen „Inseln“.

Überträgt man dieses Begriffsverständnis auf virtuelle Räume, so ist es nahe liegend, darunter solche Räume zu verstehen, die in abbildhafter und/ oder symbolischer Form medial gestaltet sind und ähnliche Funktionalitäten aufweisen. Dies bedeutet im Einzelnen:

- Die Lernenden können „den Raum betreten“ und ihre (virtuelle) Präsenz anzeigen. Dies kann z.B. durch eine Liste zurzeit angemeldeter Benutzer oder ein Präsenz-Icon geschehen.

- Die Lernenden haben ihren individuellen virtuellen Arbeitsplatz, z.B. in Form eines mit einem Namen versehenen Ordners, in dem sie persönliche Objekte ablegen (speichern) können.

4 Eine ähnliche Charakterisierung findet sich beispielsweise bei Baumgartner et al. (2002), die zwischen einem lehrerzentrierten Ansatz mit Bereitstellung eines Lehrangebots und der Abarbeitung durch Studierende und einem lernerzentrierten Ansatz mit der Bereitstellung einer lernförderlichen Umgebung mit Explorationsmöglichkeiten nach den Zielen und Wünschen der Studierenden unterscheiden (vgl. S. 22 ff.). 
- Die Lernenden können im virtuellen Raum bestimmte Aktivitäten ausführen, z.B. auf Objekte (Texte, Bilder, Grafiken, Tondokumente) einwirken, indem sie diese neu anordnen, bearbeiten, deponieren (speichern), entfernen (löschen) oder mit anderen Objekten verknüpfen. Durch diese Aktivitäten werden Spuren erzeugt, die auf die virtuelle Präsenz verweisen. Darüber hinaus können neue Objekte erzeugt und präsentiert werden.

- Die Lernenden können mit anderen Lernenden kommunizieren und sie einzeln oder als Gruppe „ansprechen“. Dies geschieht in unterschiedlichen Codierungs- und Modalitätsformen sowie in synchroner oder asynchroner Weise, z.B. durch E-Mail, Newsgroups, Chats oder Videokonferenzen.

- Die Lernenden können an Objekten gemeinsam arbeiten und genießen dabei gleiche Rechte, z.B. bei der gemeinsamen Visualisierung eines Sachverhaltes, während andere Objekte nicht für alle zugänglich sind. Dies kann z.B. durch die Vergabe besonderer Zugriffs-, Lese- und Schreibrechte realisiert werden.

Legt man virtuellen Räumen ein solches Begriffsverständnis zugrunde, werden zunächst nur solche Angebote erfasst, die mit Raummetaphern arbeiten und entsprechende Funktionalitäten (s.o.) zu simulieren versuchen. Ich werde daher in die folgenden Überlegungen all diejenigen computerbasierten Angebote einbeziehen, die - unabhängig von einer konkreten Raummetapher - „Raum bieten“ zur Exploration, zur Information, zur Dokumentation, zur Interaktion, zur Kommunikation oder zur Kollaboration. Dies bedeutet auch, dass es sich nicht in jedem Fall um ein Online-Angebot handeln muss.

\subsection{Medienfunktionen im Lernprozess}

Mit computerbasierten Medien sind bestimmte Funktionen verbunden, die Keil-Slawik (1998) als primäre Medienfunktionen bezeichnet. Im Einzelnen nennt er:

- Kreieren (Visualisieren, Symbolisieren, Modellieren, Simulieren),

- Transportieren (Senden, Verteilen, Laden, Navigieren),

- Arrangieren (gleichzeitiges Präsentieren, räumliche Abbildung logischer Zusammenhänge) und

- Verknüpfen (physisches Verbinden, Schaffen gemeinsamer Behälter, Verweisen).

Diese Medienfunktionen sind zunächst einmal Funktionen technischer Artefakte, die im Kontext von Lehren und Lernen genutzt werden können. Sie beziehen sich auf abbildhaft und/oder symbolisch codierte Darstellungsformen, also z.B. Bilder, Filme, Texte, Grafiken, Tondokumente usw. ${ }^{5}$ Verbin-

5 Strenggenommen müsste hier noch weiter differenziert werden, da - vom technischen Artefakt aus gesehen - die Objekte keine Darstellungsformen im genannten Sinne sind, sondern zunächst nur potentielle Zeichenaspekte, die - binär codiert - maschinell verarbeitbar sind (vgl. Herzig 2002). 
det man die (primären) Medienfunktionen mit grundsätzlichen Anforderungen an Unterricht bzw. an Lehr- und Lernprozesse (vgl. Abschnitte 1, 2), so gewinnen die technischen Funktionalitäten eine didaktische Qualität - oder genauer gesagt: den Medienfunktionen wird durch die Gestalter des Lernprozesses eine didaktische Qualität zugeschrieben. So können bspw. im Zusammenhang von Kooperationen die Funktionen des Kreierens, des Arrangierens und des Verknüpfens von Objekten besonders wichtig sein, während in kommunikativen Situationen die Funktion des Transportierens und des Visualisierens im Vordergrund stehen. Insgesamt können computerbasierte Medien in Lehr- und Lernprozessen in folgenden didaktischen Funktionen genutzt werden:

- als Mittel der Präsentation von Aufgaben,

- als Informationsquelle und Lernhilfe,

- als Werkzeug oder Instrument bei Aufgabenlösungen,

- als Gegenstand von Analysen und Material für weitere eigene Verwendungen und Bearbeitungen,

- als Instrument der Planung, des Austausches und der Kooperation oder

- als Werkzeug der Speicherung und der Präsentation von Ergebnissen.

Gleichzeitig erlauben bzw. unterstützen die technischen Funktionalitäten verschiedene Lernaktivitäten, die in Lehr- und Lernprozessen ermöglicht werden sollten. Dazu zählen z.B. Aktivitäten der Recherche, des Informierens, der Gestaltung, der Problemlösung, der Beurteilung, der Kommunikation oder Kooperation. Und nicht zuletzt ist mit der Kombination von Lernaktivitäten und didaktischen Medienfunktionen immer eine bestimmte Form der Sozialität verbunden, die von der selbständigen Einzelarbeit bis hin zu betreutem Lernen in Gruppen reicht, wobei sich noch einmal reale und virtuelle (telemediale) Formen der Betreuung unterscheiden lassen.

Fasst man diese Dimensionen zusammen, so erhält man einen „Raum“, innerhalb dessen computerbasierte bzw. computerunterstützte Lehr- und Lernarrangements analytisch verortet werden können (vgl. Abb. 3). Die Skalierung dieses Raums ist dabei so offen gehalten, dass die zuvor genannten Angebotsvarianten - online oder offline, mit personaler oder telemedialer Begleitung - mit erfasst werden. 


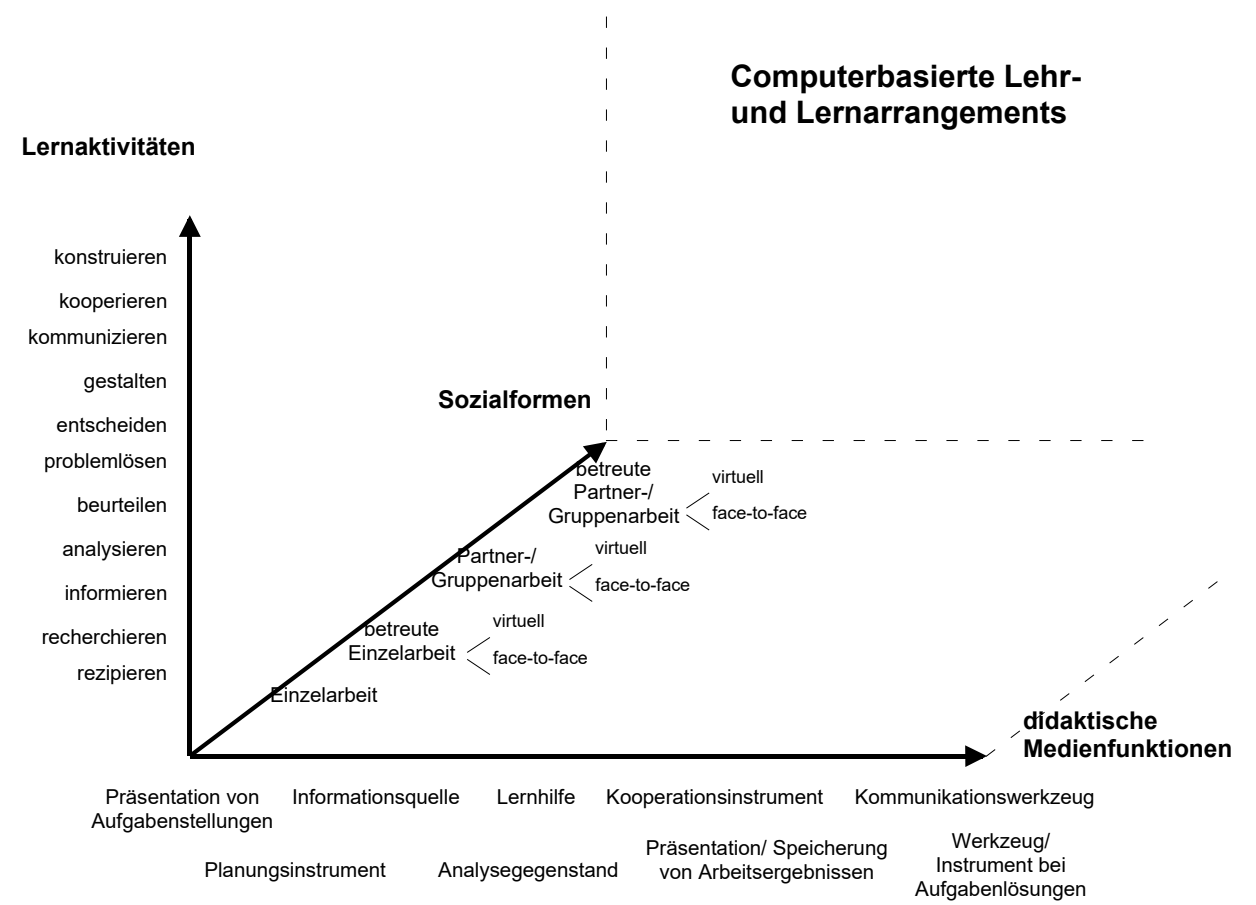

Abb. 3: „Didaktischer Raum“ zur analytischen Verortung computerbasierter Lehr- und Lernarrangements.

\section{3 „Didaktik inside“}

Ich bin in meinen Überlegungen zu didaktischen Anforderungen an Lehr- und Lernprozesse davon ausgegangen, dass Lehrhandlungen auch von Lehrsystemen bzw. Medien übernommen werden können. $\mathrm{Zu}$ solchen Lehrhandlungen zählen u.a. instruktionale Maßnahmen, die Anpassung von Lehrhandlungen an die Lernvoraussetzungen sowie die Variation und Gestaltung von Sozial- und Arbeitsformen. Im Folgenden diskutiere ich, inwieweit diese didaktischen Funktionen in computerbasierten Angeboten realisiert werden (können).

Implementation instruktionaler Komponenten in das Medienangebot

Im Zusammenhang von Forschungen zum Instruktionsdesign sind eine Reihe von Ansätzen entwickelt worden, die Aussagen darüber treffen, in welcher Folge Lehrinhalte in einzelnen Kursen oder Curricula angeordnet werden sollten und welche Schritte sinnvoll sind, damit Lernende mit bestimmten Voraussetzungen Lehrziele einer bestimmten Art erreichen (vgl. z.B. Merrill 1983, 1987; Reigeluth/ Steins 1983). Die Entwicklung medialer Produkte nach diesen Ansätzen umfasst entsprechend die Bestimmung von Lernzielen, die Identifizierung von Eigenschaften der Lernenden (im Sinne von Lernvoraussetzun- 
gen), die Auswahl und Aufbereitung der Inhalte sowie die Gestaltung von Kontrollund Feed-back-Elementen. Welche Lehrfunktion dabei konkret im jeweiligen Angebot realisiert wird, hängt vom Instruktionsmodell und der damit verbundenen lerntheoretischen Auffassung sowie den jeweils angestrebten Zielen ab. Die Umsetzungen reichen von der Darbietung kleiner Informationseinheiten mit anschließenden Kontrollfragen über strukturierte Wissenseinheiten mit Anwendungsaufgaben bis hin zu offenen Lernumgebungen mit der Präsentation von fallbasierten Aufgaben, zu denen Informationen eigenständig erarbeitet und ausgewertet werden müssen, ohne dass eine detaillierte Anweisung für einzelne Arbeitsschritte vorliegt (vgl. Merrill/ Li/ Jones 1990). Dies bedeutet auch, dass die einzelnen Instruktionsmodelle von unterschiedlich ausgeprägten Fähigkeiten zur Selbststeuerung des Lernprozesses ausgehen. Während einzelne Lehrfunktionen in lernförderlicher Weise in das Lernangebot integriert werden können - z.B. die Präsentation von Aufgabenstellungen, der Aufweis von bedeutsamen Anwendungskontexten, die Formulierung wichtiger Fragestellungen im Hinblick auf die Aufgabenlösung, die Angabe von Zielvorstellungen oder die Bereitstellung grundlegender Informationen - sind andere nur eingeschränkt zu realisieren, insbesondere wenn sie sich auf die Heterogenität von Lernenden beziehen. Dies betrifft z.B. Formen der Lernerfolgskontrolle oder der Qualitätssicherung, individuelle Rückmeldungen zum Lernprozess sowie kommunikations- und verständigungsorientierte Elemente - solange auf unidirektionale ,Kommunikations'formen zurückgegriffen wird (z.B. Aufforderungen, Anweisungen, Hilfetexte usw.).

\section{Adaption an Lernvoraussetzungen unter Nutzung (informations-)technischer Funktiona- litäten}

Geht man davon aus, dass Lernen zwar ein sozial eingebetteter, aber in hohem Maße von individuellen Eigenschaften und Voraussetzungen abhängiger Prozess ist, dann wird deutlich, dass Lehrmedien ohne personale Komponente schnell Gefahr laufen, den unterschiedlichen Voraussetzungen nicht hinreichend gerecht zu werden. Aus diesem Grunde werden computerbasierte Angebote z.T. mit technischer/ software-technischer Hilfe an die Lernvoraussetzungen adaptiert. Einfachere Formen solcher Maßnahmen beziehen sich z.B. auf die Anpassung der Lernzeit (d.h. die Frage, ob bei einem diagnostizierten Wissensstand noch weitergearbeitet oder schon ein neues Ziel angestrebt werden soll), auf die Auswahl der zu bearbeitenden Aufgaben, die Dauer ihrer Präsentation, auf die Dauer von Reaktionszeiten (die das System abwartet), auf die Anpassung von Schwierigkeitsgraden und von kontextbezogenen Hinweisen sowie von Informationen an die Interessen des Lernenden (vgl. Leutner 2002, S. 120 ff.). Darüber hinaus sind intelligente tutorielle Systeme (ITS) in der Lage, sich situativ an Lernereigenschaften anzupassen. Solche Systeme enthalten eine Wissensbasis für die Inhalte sowie ein Modul, das den jeweils aktuellen Wissensstand des Lernenden repräsentiert und In- 
formationen über Lernwege und deren Angemessenheit unter bestimmten Bedingungen enthält. Im idealen Falle sind wissensbasierte Systeme selbst lernfähig, d.h. sie verändern in der Folge ihrer „Erfahrungen“ die Lehrstrategie (vgl. Strittmatter/ Niegemann 2000, S. 135 f.).

Aus didaktischer Sicht werden mit der Adaption bzw. mit ,intelligenten’ Systemen wichtige Funktionen im Lernprozess erfasst, die sich auf die Berücksichtigung von Lernvoraussetzungen sowie auf Rückmeldungen, z.B. zum aktuellen Wissensstand, beziehen. Allerdings ist in Anbetracht der denkbaren unterschiedlichen Lernvoraussetzungen die erforderliche Komplexität bzw. Anzahl von Lernermodellen so groß, dass der mit der Entwicklung verbundene Aufwand unverhältnismäßig im Hinblick auf den zu erwartenden Erfolg erscheint. Bisher sind auch nur einzelne Funktionen von „intelligenten“ tutoriellen Systemen in Beispielen implementiert. Adaptive („unintelligente“) Maßnahmen hingegen sind leichter zu realisieren und z.B. bei Lernerfolgskontrollen oder Leistungstests in der Fehleranalyse und -diagnose bei solchen Aufgaben sinnvoll, in denen es um die Anwendung von Schemata und Regeln geht, zu denen typische Fehler bekannt sind.

\section{Kombination von Lernangeboten}

Die Implementation von instruktionalen Maßnahmen und die Anpassung von Lehrstrategien an die Lernvoraussetzungen sind stärker auf das Einzellernen ausgerichtet. Da bestimmte Phasen des Lernprozesses, insbesondere solche, die mit sozialem Austausch, Emotionalität und sozialer Nähe verbunden sind, nicht oder nicht angemessen medial simuliert werden können, ergibt sich die Notwendigkeit, verschiedene Medienangebote miteinander zu kombinieren und/ oder in Formen personaler Begleitung oder unmittelbarer sozialer Präsenz einzubetten (,hybride Angebote“).

Dazu werden telemediale Angebote z.B. mit Software-Tools kombiniert, so dass ein Austausch zwischen Lernenden (E-Mail, Chat, Newsgroups), eine Beratung durch Mentoren (Tele-Tutoren, Tele-Coaches) oder Gruppenarbeiten (workspaces mit GroupwareFunktionalitäten) möglich werden. Auch die Durchführung von Präsenzveranstaltung kann in den Phasen von Lernprozessen, in denen personale Anwesenheit eine hohe Bedeutung hat, eine angemessene Ergänzung darstellen. Formen der Lernerfolgskontrolle beispielsweise lassen sich durch Einsendeaufgaben mit individueller Rückmeldung oder in Form von Videokonferenzen durchführen.

Bei diesen Formen von Lernarrangements besteht aus didaktischer Sicht die Aufgabe, die Lernarrangements so zu gestalten, dass eine sinnvolle und lernförderliche Auseinandersetzung mit bestimmten Aufgabenstellungen möglich wird. So kann beispielsweise im Kontext eines Teleseminars eine Präsenzphase zur Präsentation der - ggf. medienunterstützten - Aufgabenstellung, zur Verständigung über Zielvorstellungen, zur Besprechung von Arbeitsschritten und -formen sowie zur Bildung von Lern- und Arbeitsgruppen genutzt werden, bevor in einer virtuellen Umgebung relevante Informa- 
tionen zusammengetragen, bearbeitet und präsentiert sowie in vergleichender Weise diskutiert werden. In der Phase der Erarbeitung können zusätzlich Offline-Medien, z.B. geeignete CD-ROMs, genutzt werden. Die individuelle Rückmeldung zu Schwierigkeiten in den individualisierten Phasen kann über Telekommunikationsdienste sichergestellt werden. Die Verbindung traditioneller Medien und präsenzgebundener Lernformen mit den besonderen Vorzügen computerbasierter Angebote wird auch unter dem Stichwort des „blended learning“ diskutiert - allerdings nicht immer auf der Basis didaktischer Überlegungen.

\section{Konsequenzen}

Die bisherigen Überlegungen machen deutlich, dass bei allen computerbasierten Angeboten die didaktische Aufgabe bestehen bleibt, diese - mit ihrer jeweiligen medieninternen didaktischen Struktur - so zu gestalten und zu verwenden, dass eine lernwirksame Wechselwirkung zwischen medieninternen Momenten (instruktionales Design, didaktische Struktur der Inhalte, Darstellungs- und Interaktionsformen usw.) und medienexternen Momenten (personale, reale Begleitung, sozialer Kontext usw.) entsteht. Je nach Angebot ist es also erforderlich, einzelne Lehrfunktionen, Kommunikations- oder Kooperationsfunktionen im Kontext des Medieneinsatzes zu planen und zu realisieren. Dies gilt in besonderem Maße für eine weitere Form computerbasierter Angebote, die in der Regel inhaltsneutral sind und Plattformen bzw. Werkzeuge zur Kooperation und Konstruktion darstellen. Sie sind Ausdruck einer zunehmenden Abkehr von produzenten- und produktzentrierten Sichtweisen und einer Hinwendung zu Formen verteilten Wissensmanagements und damit verbundener kooperativer Medien (vgl. z.B. Keil-Slawik 2001). Solche kooperativen Medien stellen technische Infrastrukturen dar, in denen virtuelle Lerngemeinschaften mit unterschiedlichen Zielsetzungen und Anforderungen realisiert werden. Entsprechende Plattformen oder virtuelle Räume stellen Kommunikations-, Dokumentenmanagements- und Koordinationsfunktionen bereit und werden von den Lernenden selbst administriert. Didaktisch gesehen, sind mit Hilfe solcher Infrastrukturen vor allem neue Formen der Erfahrung und Erkenntnisgewinnung möglich, die auf der Nutzung besonderer Medienfunktionen in Gruppen beruhen, z.B. die gemeinsame Visualisierung von Überlegungen, das Arrangieren und Verknüpfen von Objekten oder das Erzeugen neuer bzw. Verändern bestehender Objekte, verbunden mit dem ständigen Wechsel vom Autor zum Rezipienten und umgekehrt. Die didaktische „Neutralität“ solcher Angebote ist allerdings keinesfalls didaktisch folgenlos. Um erfolgreiches Lernen mit einem inhaltsneutralen Werkzeug zu ermöglichen, muss zunächst ein Lernprozess angeregt, initiiert und dann - personal oder medial - unterstützt und begleitet werden. Mit anderen Worten: Auch Lernprozesse auf sogenannten Lernplattformen sind an bedeutsame Aufgabenstellungen, an aufgabenrelevante Materialien oder an gemeinsame Zielvereinbarungen gebunden. Einen solchen didakti- 
schen Kontext herzustellen, ist im Falle dieser Systeme in zweifacher Hinsicht wichtig: Zum einen geht es um die Integration eines Mediums in einen Lehr-Lernprozess, zum anderen um die didaktische Gestaltung des Mediums im Rahmen dieses Lehr-Lernprozesses selber, z.B. die Gestaltung virtueller Räume mit Dokumenten, Gruppen, Rechten usw.

Damit ist auch noch einmal betont, dass Medien als technische Artefakte einzelne Lernphasen bzw. die damit zusammenhängenden Denkprozesse in lernförderlicher Weise unterstützen können, aber weder Organisatoren von Lernprozessen sind noch automatisch eine besondere Qualität von Lernprozessen sicherstellen. Eine solche Qualität - sowohl im Bezug auf den Prozess als auch auf die erreichten Lernziele - wird im Wesentlichen davon abhängen, inwieweit es gelingt, eine handlungs- und entwicklungsfördernde Auseinandersetzung des Einzelnen bzw. von Gruppen mit bedeutsamen Aufgabenstellungen anzuregen und zu unterstützen.

\subsection{Empirische Aspekte}

\section{Medienbezogene Lehr-Lernforschung}

Die Annahme, die Qualität eines Lernprozesses werde nicht durch die Art des Mediums, sondern stärker durch die didaktische Gestaltung des gesamten Lehr- und Lernarrangements beeinflusst, kann auch durch empirische Überlegungen untermauert werden. Die Lehr-Lernforschung im Kontext von Medien lässt sich grob in vier Richtungen unterscheiden:

- Untersuchungen zu allgemeinen Medieneffekten,

- Untersuchungen zu speziellen Medienmerkmalen,

- interaktionsorientierte Studien und

- Evaluationen.

Die Untersuchungen zu generellen Medieneffekten (z.B. computerbasiertes Lernen vs. Lernen in herkömmlicher Weise) zeigen insgesamt keine einheitlichen Effekte; sie bewegen sich zum großen Teil im „Land der Nullhypothesen“ (Schulmeister 2002, S. 387) und werden heute nicht mehr als sinnvolles Forschungsdesign betrachtet. Entsprechende Metastudien haben nur in einzelnen Aspekten relevante Ergebnisse (z.B. zur Verkürzung der Lernzeit) gezeigt, Clark (1994) formulierte angesichts der Forschungslage sogar: „media will never influence learning“. Nicht zuletzt ist die Konfudierung von didaktischer Gestaltung und technischem Medium als ein wesentlicher Kritikpunkt an Studien zu generellen Medienvergleichen angeführt worden.

Untersuchungen zu speziellen Medienmerkmalen fragen danach, wie bestimmte Medieneigenschaften sich auf den Lernerfolg - i. d. R. den Wissenserwerb - auswirken. Auch hierzu gibt es inzwischen eine Vielzahl von experimentellen Studien, die zum großen Teil theoriegeleitet sind. Im Hinblick auf das Lernen mit multimedialen Materialien ist die generative Theorie 
multimedialen Lernens von Mayer (1997) bzw. Mayer/ Moreno (1998) die heute aussagkräftigste und empirisch gut bestätigte Theorie. Sie erlaubt Aussagen darüber, in welcher Weise textliche, bildliche und auditive Informationen präsentiert werden sollten, um den Wissenserwerb zu optimieren. Die aus diesem Ansatz abgeleiteten Gestaltungsprinzipien finden inzwischen breite Verwendung.

Interaktionsstudien gehen davon aus, dass Medieneffekte eine Wechselwirkung zwischen den individuellen Lernvoraussetzungen und Medienmerkmalen darstellen. Die Voraussetzungen beziehen sich z.B. auf bereichsspezifisches Vorwissen, auf besondere Einstellungen (z.B. gegenüber neuen Technologien) oder auf übergreifende Persönlichkeitsmerkmale, z.B. das räumliche Vorstellungsvermögen. Darüber hinaus haben sich medienspezifisches Wissen, Motivation und Lernstrategien als relevante Einflussfaktoren erwiesen.

All die genannten Forschungen sind letztlich notwendige, aber nicht hinreichende Voraussetzungen für eine umfassendere Lehr-Lernforschung, die Auskunft über die Lernwirksamkeit von Medien in Lehr-Lernprozessen geben kann. Die Vielfalt der Einflussfaktoren und die Komplexität von Lehr-Lernprozessen ist über experimentelle Studien in der Regel nicht zu erfassen. Dennoch liefern sie wichtige Erkenntnisse zu einzelnen Aspekten, wie z.B. die generative Theorie multimedialen Lernens zeigt. Ein weiterführendes Forschungsdesign könnten hier Evaluationsstudien bieten, die - im Vergleich zur experimentellen Hypothesenprüfung (erkenntnisorientiert) - eher als entscheidungsorientiertes Verfahren zur Verbesserung und Überprüfung der Wirksamkeit von Maßnahmen charakterisiert werden können (vgl. z.B. Tulodziecki 1982; Herzig 1998). Ein solches Forschungsdesign geht von vornherein von einem größeren Kontext aus und prüft auf der Basis von Ziel-Mittel-Aussagen, ob sich ein bestimmtes Mittel im Hinblick auf eine gegebene Zielvorstellung als bewährt erwiesen hat und welche Nebenwirkungen ggf. festzustellen sind. Dies bedeutet, dass theoretische Annahmen nicht auf ihre „Richtigkeit“ hin, sondern auf ihre Anwendbarkeit hin geprüft werden.

\section{Besondere Problemlagen in virtuellen Räumen}

Ich habe bei der Charakterisierung von Medienangeboten darauf hingewiesen, dass beim Übergang von realen zu virtuellen Räumen verschiedene Aspekte der sozialen Präsenz nicht vollständig kompensiert werden können. Gerade bei (kooperativen) Lernangeboten in virtuellen Umgebungen (i.d.F. vollvirtuelle Online-Plattformen), die nicht in soziale Präsenzphasen eingebettet sind, können vergleichsweise hohe Ausstiegsraten beobachtet werden (dropout) (vgl. Moore/Thompson 1997). Hinzu kommen Problemlagen, wie z.B. übermäßiges Beitragsverhalten (bis hin zum spamming), unzureichendes Beitragsverhalten auf Kosten der Kooperationspartner (social loafing) oder Schwierigkeiten, innerhalb eines geteilten Bezugsrahmens (common ground) eine Verständigung zu erreichen (social grounding). In diesem Zusammenhang weisen Zumbach/Reinmann (2003) darauf hin, dass die Mitglieder einer 
Gruppe davon überzeugt sein müssen, „dass sie ein Ziel erreichen können und dies vor allen Dingen davon abhängt, dass alle in der Gruppe dieses Ziel erreichen müssen. Dadurch werden positive Abhängigkeiten geschaffen, die das Teilen von Wissen und Ressourcen, echte Kollaboration und die Bildung einer Gruppenidentität notwendig machen“ (S. 10). Mit Bezug auf die allgemein didaktischen Anforderungen an Lernprozesse wird hier noch einmal der Stellenwert einer geeigneten Aufgabenstellung deutlich, die bei den Lernenden (gemeinsame oder auch unterschiedliche) Bedürfnisse ansprechen sollte, die für gegenwärtiges oder zukünftiges Handeln der Kooperierenden bedeutsam sein sollte und die einen angemessen Schwierigkeitsgrad aufweisen sollte. In kooperativen Lernprozessen wird darüber hinaus die Anforderung an die Aufgabe gestellt, dass sie die gemeinsame Erarbeitung einer Aufgabenlösung erfordern muss. Ist die Aufgabenstellung so gehalten, dass sie eine individuelle Bewältigung zulässt, ist die Kollaboration - so sie dann überhaupt stattfindet - eher eine künstliche. Insofern zeigt sich hier noch einmal, wie wichtig auf der einen Seite eine besondere Aufgabenkultur ist, und wie wichtig es auf der anderen Seite ist, die Auswahl des Mediums von der Zielstellung her zu begründen, nicht das Medium selbst zum Ausgangspunkt der Überlegungen zu machen. Den erfolgreichen Aufbau funktionierender Gruppen sehen Zumbach/ Reimann an die geregelte Kommunikation zwischen allen Lernenden (Beachtung grundlegender Kommunikationsregeln) und die erfolgreiche Unterstützung kollaborativer Prozesse durch einen Tutor (vgl. S. 19) gebunden. Die effektive Nutzung von Medienkanälen hängt wiederum davon ab, „wie ihre Merkmale mit aufgabenrelevanten Kommunikationsprozessen interagieren“ (S. 20). Weitere Maßnahmen zur Unterstützung von Kollaborationsprozessen auf die hier nicht näher eingegangen werden kann - sind beispielsweise Werkzeuge zur gemeinsamen Visualisierung oder Kooperationsskripts (vgl. z.B. Weinberger/ Fischer/ Mandl 2002).

Im Hinblick auf die Forschung zu kollaborativen Lernumgebungen kommen Zumbach / Reimann zu dem folgenden Ergebnis: „Bestrebungen, Aussagen der Form „Technologie X führt zu Problemen Y, die durch Maßnahme Z behoben/ kompensiert werden können“ treffen zu können, sind auf Dauer nicht unbedingt fruchtbar. Vielmehr ist immer mit Interaktionen von Technologien, Personen- und Inhaltsmerkmalen, Aufgabenstellungen und Lernzielen zu rechnen“ (2003, S. 19). Forschungsmethodisch betont dies noch einmal den Stellenwert von evaluativen Studien (s.o.) unter stärker allgemein didaktischen Fragestellungen - allerdings nicht in Konkurrenz zu mediendidaktischen oder medienpsychologischen Ansätzen, sondern als Integration und Ergänzung. Abschließend beziehe ich die bisherigen Überlegungen in einzelnen Punkten auf den Fernstudiengang Medien (FESTUM). 


\section{Fernstudium Medien (FESTUM) - Didaktische Anmerkungen}

\section{Ausgangslage und curriculare Konzeption}

Auf der Basis verschiedener Projekte zur Lehrerausbildung im Medienbereich an den Universitäten Paderborn (BIG - Bildungswege in der Informationsgesellschaft), Dortmund (IKARUS - Informations- und Kommunikationstechnologische Ausbildung im Rahmen des Lehramts-Studiums“) und Bielefeld (MeKoLa - Medienkompetenz in der Lehrerausbildung) wurde 1999 an der Universität Paderborn ein Zusatzstudiengang „Medien und Informationstechnologien in Erziehung, Unterricht und Bildung“ genehmigt und bietet seither den Lehramtsstudierenden die Möglichkeit, medienpädagogische Kompetenz in einem Zusatzangebot zu erweitern und mit einer Staatsprüfung abzuschließen ${ }^{6}$.

Um das Angebot nicht lokal zu beschränken und auch Lehrerinnen und Lehrern im Vorbereitungs- und im Schuldienst sowie Absolventinnen und Absolventen eines Hochschulstudiums, die in affinen Berufsfeldern tätig sind, den Zugang zu ermöglichen, wurde im Juli 2000 das Projekt FESTUM zur Entwicklung eines Fernstudiengangs für den Erwerb der Zusatzqualifikation an der FernUniversität Hagen bewilligt und im Sommersemester 2001 mit einer zweijährigen Pilotphase gestartet, die im März 2003 ausläuft. ${ }^{7}$

Die inhaltliche Ausgestaltung des Angebots orientiert sich zum einen an den schulischen Aufgaben im Medienbereich, zum anderen an den erforderlichen medienpädagogischen Kompetenzen der Lehrpersonen. Die curriculare Ausrichtung des Studiengangs berücksichtigt sämtliche Aspekte von Mediennutzung, Mediengestaltung und unterrichtlichem Medieneinsatz bis hin zu Fragen der Schul- und Curriculumentwicklung (vgl. Abb. 4).

\section{Didaktische Strukturierung des Angebots}

Der weiterbildende Studiengang Medien (FESTUM) ist als Fernstudiengang dergestalt konzipiert, dass Studierende im Wesentlichen in Einzelarbeit und ohne räumliche oder zeitliche Bindung mit dem Studienmaterial arbeiten können. Möglichkeiten zum bedarfsorientierten, individuellen und flexiblen Arbeiten lassen auf der einen Seite Anpassungen an die individuellen Lebensumstände zu, erfordern aber auf der anderen Seite eine besondere didaktische Strukturierung und Konzeption des Studienangebots.

6 Eine entsprechende Zusatzqualifikation wird inzwischen auch von den Universitäten Münster und Bielefeld angeboten.

7 Zurzeit befindet sich der Studiengang im Akkreditierungsverfahren zum weiterbildenden Studiengang der FernUniversität mit einem Masterabschluss und dem damit verbundenen Titel „Master of Arts in Media Education“. 
Die Materialien zum Fernstudium Medien bestehen aus einzelnen Kurseinheiten zu den in Abbildung 4 genannten Themenbereichen, die in netzbasierter Form, auf CD-ROMs oder auch als Studienbriefe verfügbar sind. Die online verfügbaren Angebote sind über den Lernraum Virtuelle Universität zugänglich. Die Betreuung der Studierenden erfolgt bislang telefonisch, über Mailing-Listen, Newsgroups, und in Foren. Die technische Plattform ermöglicht darüber hinaus den Austausch der Studierenden untereinander zu inhaltlichen Fragen im Zusammenhang mit den Kurseinheiten, zu Fragen der Studienorganisation, zu Klausuren und Seminararbeiten, zum Praktikum oder zu Prüfungen.

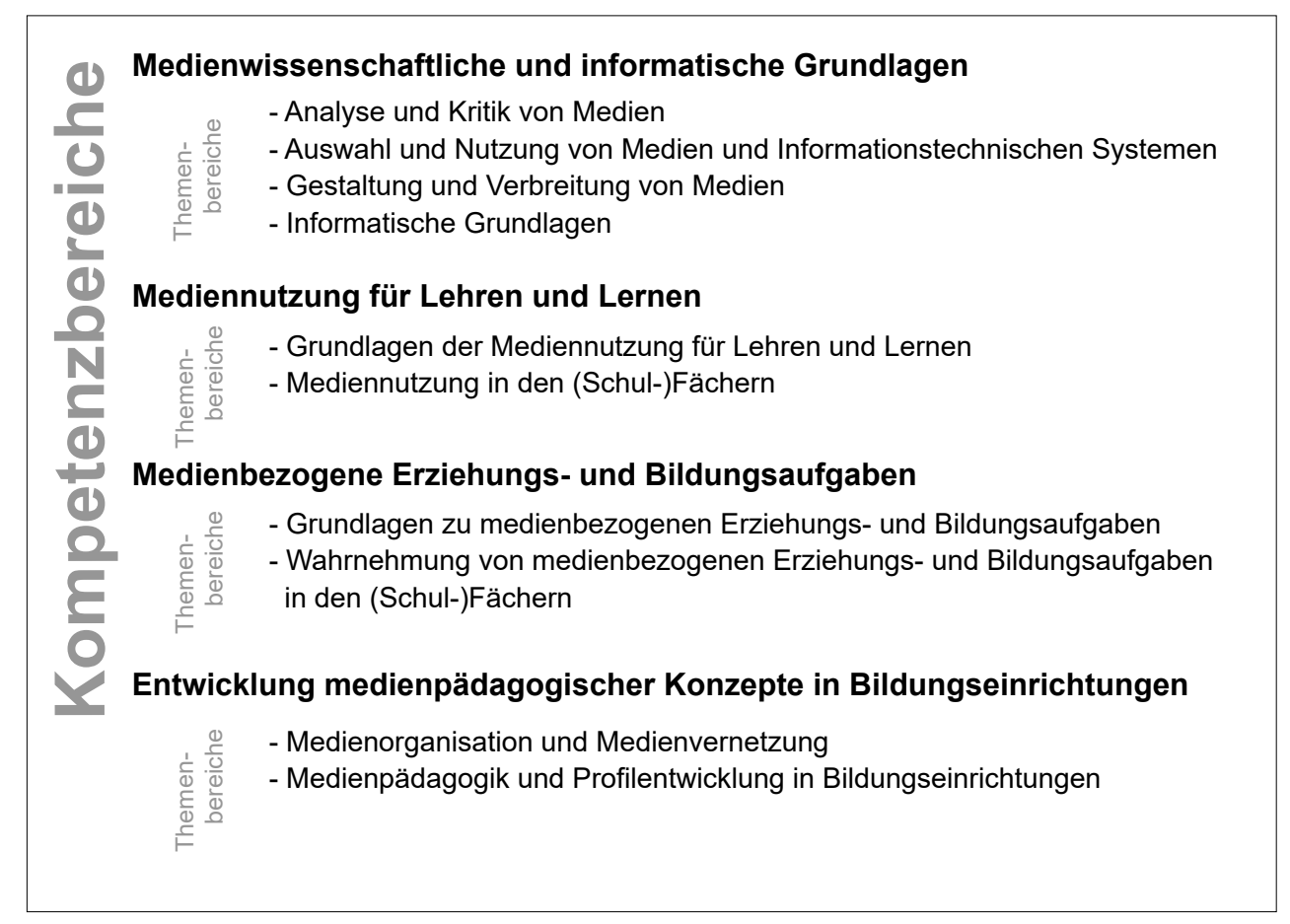

Abb. 4: Kompetenz- und Themenbereiche des FESTUM-Curriculum.

Der Aufbau der einzelnen Kurseinheiten folgt dem - im vorliegenden Beitrag skizzierten - handlungsorientierten didaktischen Ansatz, der davon ausgeht, dass Lernprozesse durch bedeutsame Aufgabenstellungen angeregt werden können. Solche Aufgaben bilden den Ausgangspunkt der einzelnen Kurseinheiten und sind als Problemstellungen, Entscheidungsfälle, Beurteilungsfälle oder Gestaltungsaufgaben formuliert. Inhaltlich erfassen die Aufgaben solche Handlungssituationen, wie sie für Lehrpersonen im Medienbereich relevant sind. Die Auseinandersetzung mit dem Studienmaterial erfolgt damit nicht losgelöst von möglichen späteren Handlungsfeldern, sondern setzt eben hier an und ermöglicht damit den Studierenden, sich zunächst einmal über die noch notwendigen Kenntnisse und Fertigkeiten bewusst zu werden, die erforderlich sind, um die entsprechende Aufgabe zu lösen. In den so genannten 
grundlegenden Informationen können diese fehlenden Fähigkeiten und Wissensbestände dann erarbeitet und anschließend auf die Aufgabenstellung angewendet werden. Der Vergleich der eigenen Lösung mit einem Lösungskommentar bietet eine Form der Erfolgskontrolle. Einzelne Aufgaben sind darüber hinaus als Einsendeaufgaben formuliert und werden mit einem individuellen Kommentar bzw. einer Beurteilung an die Studierenden zurückgesendet. Erfolgskontrollen sind ferner durch studienbegleitende Klausuren und Hausarbeiten gegeben. Zur Vertiefung und/ oder Ergänzung der Inhalte finden sich am Ende einer Kurseinheit jeweils kommentierte Literaturempfehlungen und Hinweise auf relevante Internetadressen. Die Vernetzung der einzelnen Kurseinheiten bzw. ihrer Inhalte wird durch ein Online-Glossar unterstützt.

Vor dem Hintergrund der Überlegungen im vierten Abschnitt ist das Angebot als ein Lehr-Lernarrangement einzuordnen, in dem in Einzelarbeit und (virtuell) betreuter Einzelarbeit verschiedene Lernaktivitäten (insbesondere rezipieren, recherchieren, informieren, analysieren, beurteilen, problemlösen, entscheiden, gestalten) auf der Basis verschiedener Medienangebote (Studienbrief, CD, Online-Kurs) durchgeführt werden. Von den Medienangeboten werden dabei vor allem folgende Funktionen wahrgenommen:

- Präsentation von Aufgabenstellungen, Informationsquelle, Lernhilfe, Analysegegenstand (Studienbrief, CD, Online-Kurs)

- Kommunikationswerkzeug (Online-Plattform).

\section{Erste Erfahrungen und Problemlagen}

Umfassende Ergebnisse zur Pilotphase des Projekts liegen im Moment noch nicht vor, die abschließende Befragung wird im Moment durchgeführt. Erste Hinweise auf Problemlagen und mögliche Perspektiven lassen sich aber aus einer Befragung von Studienabbrechern und Studierenden der Pilotphase entnehmen (vgl. Weritz 2003) sowie aus den Logfiles von Chat-Sitzungen und aus den Newsgroups.

Versucht man zunächst die Gründe für den Studienabbruch ${ }^{8}$ zu klassifizieren, so stechen zwei Kategorien hervor (hier sind nur die Studierenden berücksichtigt, die angeben, dass der Studienabbruch maßgeblich mit dem Studium zu tun hat):

8 Die Abbrecherquote betrug im ersten Semester ca. 23\%, im zweiten Semester ca. 18\%. Darunter sind - insbesondere im ersten Semester - allerdings eine Vielzahl von Studierenden, die sich zunächst fristwahrend eingeschrieben haben und dann - nach Einsicht in die Studienunterlagen sowie genauerer Kenntnis der Zielsetzungen und Inhalte - wieder ausgestiegen sind. Zudem ist zu berücksichtigen, dass Studienabbruch nicht immer in unmittelbarem Zusammenhang mit dem Studium selbst steht. Von den befragten Studienabbrechern gaben 56\% an, dass die Aufgabe des Studiums nichts oder nur wenig mit dem Studium selbst zu tun haben. 
- nicht unmittelbar in die schulische Praxis umsetzbare Studieninhalte (zu geringe Praxisrelevanz),

- zu hoher zeitlicher Aufwand.

Der hohe Zeitaufwand ist in der Regel eine Frage der persönlichen Lebensumstände (Beruf und Studium parallel) und didaktisch weniger bedeutsam, wenn man unterstellt, dass der Aufwand nicht durch unverständliche oder übermäßig schwierig zu erschließende Inhalte verursacht wird (s.u.). Die geringe Praxisrelevanz hingegen ist didaktisch bedeutsam, weil von der Einschätzung der Praxisrelevanz der zu erarbeitenden Inhalte auch die subjektiv empfundene Bedeutsamkeit und damit die Motivation abhängen (vgl. Abschn.e 1 und 2). Sie lässt sich in zweierlei Hinsicht deuten: zum einen kann es um die Frage der Umsetzung von der Theorie in die schulische Alltagspraxis gehen, zum anderen um die grundsätzliche Bedeutsamkeit der behandelten Fragestellungen für die Medienpädagogik in der Schule. Beide Varianten spielen für die Studienabbrecher eine Rolle.

Zum einen scheint sich ein Theorie-Praxis-Verständnis abzuzeichnen, das stärker einer technologischen Transfermetapher verhaftet ist und dementsprechend den Stellenwert theoretischer Überlegungen - die Bestandteil aller Studieninhalte sind - eher geringer erscheinen lassen. Zum anderen kann vermutet werden, dass das individuelle Erleben schulischen Alltags und schulischer Aufgaben im Medienbereich als Messlatte für die Realitätsnähe von Aufgabenstellungen oder Fallbeispielen herangezogen wird - und damit u.U. auch die Bereitschaft verringert, sich auf solche Situationen als grundsätzlich wichtige und bedeutsame Situationen einzulassen, die im schulischen Alltag bisher nicht erlebt wurden.

Diese Vermutungen müssen in der weiteren Evaluation geprüft werden, im Moment stellen sie Hypothesen auf der Basis der Befragung einzelner Studienabbrecher dar. Insbesondere sind sie mit den Erfahrungen und Einschätzungen der aktiven Studierenden zu vergleichen. Deutlich ist aber in jedem Fall, dass dieses Problems kein medienbezogenes, sondern ein didaktisches ist. Ein gewisses „Dilemma“ könnte sich hier abzeichnen zwischen den Bedürfnissen der Klientel nach möglichst alltagspraktischen Anregungen für die Unterrichtspraxis und den Ansprüchen, die an ein wissenschaftliches Studium gestellt werden. Aber auch dies ist keineswegs neu, sondern eine in der Professionalisierungsdebatte in der Lehrerausbildung seit langem diskutierte Problemlage, die bei Lehrpersonen mit langer Berufspraxis u.U. noch einmal eine besondere Akzentuierung erhält. Mit Bezug auf das in Abschnitt 2 dargestellte Lern- und Handlungsmodell lassen sich diese Ergebnisse aber auch so deuten, dass das Lernen in handlungsorientierten Kontexten von einigen grundsätzlich in Frage gestellt wird (was nicht gleich bedeutend mit ablehnen ist), weil die Kontexte selbst aus verschiedenen - noch zu ermittelnden Gründen als nicht angemessen oder sinnvoll angesehen werden. Ein solcher Lernprozess ist in jeder Hinsicht wünschenswert, wirft allerdings die nicht triviale Frage auf, wie er im Rahmen eines Fernstudiums moderiert werden kann. 
Ein weiterer - didaktisch bedeutsamer Effekt - zeigt sich in den Anmerkungen zum Aufbau der Studienmaterialien: Die Qualität der Studienmaterialien wurde von den befragten Studienabbrechern in Bezug auf die Inhalte im Spektrum von 2,0 bis 3,0 und in Bezug auf die Verständlichkeit im Spektrum von 1,8 bis 3,0 je nach zugrunde liegender Kurseinheit beurteilt (zugrundeliegende Notenskala von 1 bis 6). Der didaktische Aufbau der Materialien (einleitende Hinweise, Lernziele, Aufgabenstellung/ Fall, grundlegende Informationen, ggf. weiterführende Informationen, Lösungskommentar, kommentierte Literaturhinweise, Internetverweise) wurde von 53\% der Befragten als erleichternd für das Studium eingeschätzt, 7\% empfanden ihn als erschwerend, $40 \%$ als weder erleichternd, noch erschwerend. Dieses Ergebnis trifft auch für die Studierenden des Pilotstudiengangs zu. Trotz dieser insgesamt eher positiven Einschätzung der allgemein didaktischen Struktur, zeigte sich in einzelnen freien Antworten, dass offenbar das Vorverständnis von Didaktik (im Sinne subjektiver Theorien über die Gestaltung von Unterricht) bzw. die eigene didaktische Vorgehensweise im Unterricht nicht unerheblichen Einfluss auf die Auseinandersetzung mit den - der eigenen Auffassung entsprechenden oder auch widersprechenden - didaktisch gestalteten Materialien hat. Auch dieser Einfluss ist in der weiteren Evaluation zu prüfen - an dieser Stelle ist zunächst festzuhalten, dass es ebenfalls kein medienbezogenes, sondern ein originär didaktisches Problem ist.

Ergänzend sei darauf hingewiesen, dass die mediendidaktische Gestaltung der Online-Kurse, insbesondere die interaktiven Elemente, nach den ersten Rückmeldungen aus den Newsgroups und Chat-Sitzungen positiv aufgenommen werden. Dies gilt insbesondere für animierte Darstellungen, in denen dynamische Prozesse simuliert werden. Ich werde darauf an dieser Stelle nicht näher eingehen, weil dies sich auf im engeren Sinne mediendidaktische Aspekte bezieht.

Die Online-Plattform, auf der ein Teil der FESTUM-Kurse bereitgestellt ist, bietet außerdem die Möglichkeit zur Kommunikation und Kooperation. Diese Möglichkeiten werden allerdings nur äußerst selten genutzt. Die Inhalte der Chats (u.a. als OnlineSprechstunde) und der Newsgroups beziehen sich häufig auf organisatorische Fragen (z.B. Prüfungsregelungen, Termine, Klausurthemen, Studienmaterialien usw.), nur in vereinzelten Fällen auf inhaltliche Aspekte der Studienmaterialien, für die gesonderte kursbezogene Foren eingerichtet sind. Allerdings hat die geringe Nutzung der Foren dazu geführt, dass die - wenigen - Teilnehmerinnen und Teilnehmer genau dies zum Thema in Chats gemacht haben.

Didaktisch interessant ist hierbei vor allem der Hinweis, dass die Teamarbeit durch eine entsprechende Aufgabenstellung motiviert sein muss. Kooperation um der Kooperation willen birgt die Gefahr der oberflächlichen, unkonstanten oder auch frustrierenden „Team“arbeit. Für den Fernstudiengang FESTUM sind die Materialien so angelegt, dass eine eigenständige Bearbeitung mit einem Austausch über Inhalte sinnvoll und angemessen ist, „echte“ kooperative Aufgaben, die nur in Kooperation mit anderen zu lösen 
sind, sind nicht vorhanden. Nichtsdestotrotz bleibt zu überlegen, ob nicht gerade durch kooperative Aufgaben in den Studienmaterialien eine inhaltliche Notwendigkeit für Kooperation erzeugt werden kann/ sollte, um darüber ggf. auch den stärkeren - nicht aufgabenbezogenen - Austausch anzuregen, wenn sich kleine Arbeitsgruppen gebildet haben.

\section{Literatur}

Baumgartner, P./ Häfele, H./ Maier-Häfele, K.: E-Learning Praxishandbuch. Auswahl von Lernplattformen. Innsbruck: Studienverlag 2002.

$B L K$ - Bund-Länder-Kommission für Bildungsplanung und Forschungsförderung: Medienerziehung in der Schule. Orientierungsrahmen. Bonn: BLK 1995.

Bunk, G. P./ Kaiser, M./ Zedler, R.: Schlüsselqualifikationen - Intention, Modifikation und Realisation in der beruflichen Aus- und Weiterbildung. Mitteilungen aus der Arbeitsmarkt- und Berufsforschung. 24 (1991) 2, S. $365-374$.

Gilligan, C.: Die andere Stimme. Lebenskonflikte und Moral der Frau. 5. Aufl., München: Piper 1991.

Gudjons, H.: Handlungsorientiert lehren und lernen. Dritte Aufl., Bad Heilbrunn 1992.

Heimann, P.: Didaktik als Theorie und Lehre. Die Deutsche Schule 54(1962)9, S. 409-427.

Herzig, B.: Förderung ethischer Urteils- und Orientierungsfähigkeit. Grundlagen und schulische Anwendungen. Münster u.a. 1998.

Herzig, B.: Analoge und digitale Medien im Bildungsprozess. Theoriebasierte Entwicklung einer integrativen Sichtweise für die Medienbildung. Habilitationsschrift, Paderborn: Universität, Fakultät für Kulturwissenschaften.

Jank, W./ Meyer, H.: Didaktische Modelle. Frankfurt a.M. 1991.

Keil-Slawik, R.: Anmerkungen zu einem kulturell unbewältigten Verhältnis. In: Arbeitsgemeinschaft betriebliche Weiterbildungsforschung (Hrsg.): Arbeiten und Lernen. Lernen, Kompetenzentwicklung und innovative Arbeitsgestaltung. Berlin: QUEM-report, Heft 67, S. 109-118.

Keil-Slawik, R./ Selke, H.: Forschungsstand und Entwicklungsperspektiven zum virtuellen Lernen von Erwachsenen. [http://iug.uni-paderborn.de/iug/veroeffentlichungen/1998/rks_hase_kompetenz] (02/2003).

Kohlberg, L.: Zur kognitiven Entwicklung des Kindes. Frankfurt a.M. 1974.

Kohlberg, L.: Kognitive Entwicklung und moralische Erziehung. Politische Didaktik 3 (1977) 1, S. $5-21$.

Leutner, D.: Adaptivität und Adaptierbarkeit multimedialer Lehr- und Informationssysteme. In: Issing, L. J./ Klimsa, P. (Hrsg.): Information und Lernen mit Multimedia und Internet. Weinheim 2002, S. 115-125.

Mandl, H./ Gruber, H./ Renkl, A.: Situiertes Lernen in multimedialen Lernumgebungen. In: Issing, L./ Klimsa, P. (Hrsg.): Information und Lernen mit Multimedia und Internet. Weinheim 2002, S. 139-148.

Mayer, R. E.: Multimedia learning: Are we asking the right questions? Educational Psychologist 32(1997), S. 1-19.

Mayer, R. E./ Moreno, R.: A split-attention effect in multimedia learning: evidence for dual processing systems in working memory. Journal of Educational Psychology 90(1998), S. 312-320.

Merril, M. D.: Component Display Theory. In: Reigeluth, C. M. (Hrsg.): a.a.O., S. 335-382. 
Merrill, M. D.: The New Component Display Theory: instructional Design for Courseware Authoring. Instructional Science 16(1987)1, S. 19-34.

Merrill, M. D./ Li, Z./ Jones, M. K.: Second generation instructional design (ID2). Educational Technology 31(1990)6, S. 7-12.

Möller, C.: Technik der Lernplanung. Methoden und Probleme der Lernzielerstellung. Weinheim, Basel 1973.

Moore, M. G./ Thompson, M. M.: The effects of distance learning. University Park, PA: American Center for the Study of Distance Education 1997.

Reigeluth, C. M./ Steins, F. S.: The elaboration theory of instruction. In: Reigeluth, C.M. (Hrsg.): a.a.O., S. 279-333.

Schnotz, D.: Zum Thema Instruktionsdesign: Typen versus Dimensionen. [http://www.iwm-kmrc.de/kevih/workshops/didaktikmat/SchnotzkevihWS.pdf] (02/2003).

Schroder, H.M./ Driver, M.J./ Streufert, S.: Menschliche Informationsverarbeitung. Die Strukturen der Informationsverarbeitung bei Einzelpersonen und Gruppen in komplexen sozialen Situationen. Weinheim 1975.

Schulmeister, R.: Grundlagen hypermedialer Lernsysteme. München u.a. 2002.

Schulz, W.: Unterrichtsplanung. Dritte Aufl., München 1981.

Streufert, S./ Streufert, S.: Behavior in the Complex Environment. New York 1978.

Strittmatter, P./ Niegemann, H.: Lehren und Lernen mit Medien. Darmstadt 2000.

Tulodziecki, G.: Zur Bedeutung von Erhebung, Experiment und Evaluation für die Unterrichtswissenschaft. Unterrichtswissenschaft (1982) 10, S. 364-377.

Tulodziecki, G.: Medienerziehung in der Schule - Zielsetzungen, Strategien, Methoden. In: Hamm, I. (Hrsg.): Medien als Bildungsaufgabe in Ost und West. Gütersloh 1993, S. 59-66.

Tulodziecki, G.: Unterricht mit Jugendlichen. Eine handlungsorientierte Didaktik mit Unterrichtsbeispielen. Bad Heilbrunn 1996.

Tulodziecki, G.: Medien in Erziehung und Bildung. Grundlagen und Beispiele einer handlungs- und entwicklungsorientierten Medienpädagogik. 3. Aufl., Bad Heilbrunn 1997.

Weinberger, A./ Fischer, F./ Mandl, H.: Gemeinsame Wissenskonstruktion in computervermittelter Kommunikation: Welche Kooperationsskripts fördern Partizipation und anwendungsorientiertes Wissen? (Forschungsbericht Nr. 153) München: Ludwig-Maximilians-Universität, Lehrstuhl für Empirische Pädagogik und Pädagogische Psychologie 2002.

Weritz, W.: Auswertung der Befragung von Studienabbrechern des Fernstudiengangs Medien (FESTUM). Arbeitspapier, Fakultät für Kulturwissenschaften: Universität Paderborn 2003.

Zumbach, J./ Reimann, P.: Analyse und Förderung komplexer Kooperation und Kollaboration in synchronen Lernumgebungen. [http://paeps.psi.uni-heidelberg.de/wissensbildung/downloads/ analyse01.pdf] (02/2003). 